\title{
GOLD-Empfehlungen im Praxistest
}

\author{
_ Die GOLD-Empfehlungen werden im \\ Praxisalltag nur teilweise umgesetzt - so \\ das Ergebnis einer deutschlandweiten \\ Erhebung zum COPD-Management mit \\ 451 niedergelassenen Pneumologen \\ [PneumoNews. 2015;7(5):53-57]. Als \\ Grund dafür wird die fehlende Prakti- \\ kabilität der Empfehlungen angegeben. \\ An der aktuellen GOLD-Klassifika- \\ tion zur Einordnung einer COPD orien- \\ tieren sich $41,5 \%$ der Teilnehmer nicht. \\ 37,3\% monierten u.a., dass die Erfas- \\ sungsmöglichkeiten mittels Fragebögen/ \\ Scores nicht ausreichend bzw. nicht \\ praktikabel seien. \\ Gefragt wurde auch nach möglichen \\ Gründen für eine in der Patientenwahr- \\ nehmung morgens deutlicher ausge- \\ prägte COPD-Symptomatik: Hier wur-
}

den am häufigsten Sekretstau durch einen reduzierten Hustenreflex in der Nacht $(88,9 \%)$ und ein erhöhter Parasympathikotonus in der Nacht (59,0\%) genannt.

Untersuchte man die Therapie, zeigte sich unter anderem, dass LAMA/ LABA-Kombinationen (etwa Brimica ${ }^{\circ}$ Genuair $^{\circ}$ [400 $\mu \mathrm{g}$ Aclidiniumbromid plus $12 \mu \mathrm{g}$ Formoterolfumarat]) bereits regelmäßig und abhängig vom Schweregrad eingesetzt werden: Im Mittel bei $33,8 \%$ der Patientengruppe $\mathrm{B}$ nach GOLD, bei 79,9\% der Gruppe $C$ und bei 92,9\% der Gruppe D.

Für die Therapieadhärenz sehen $83,6 \%$ die langfristige Verträglichkeit der Medikation als wesentliche Einflussgröße. Nach Einschätzung von 96,7\%

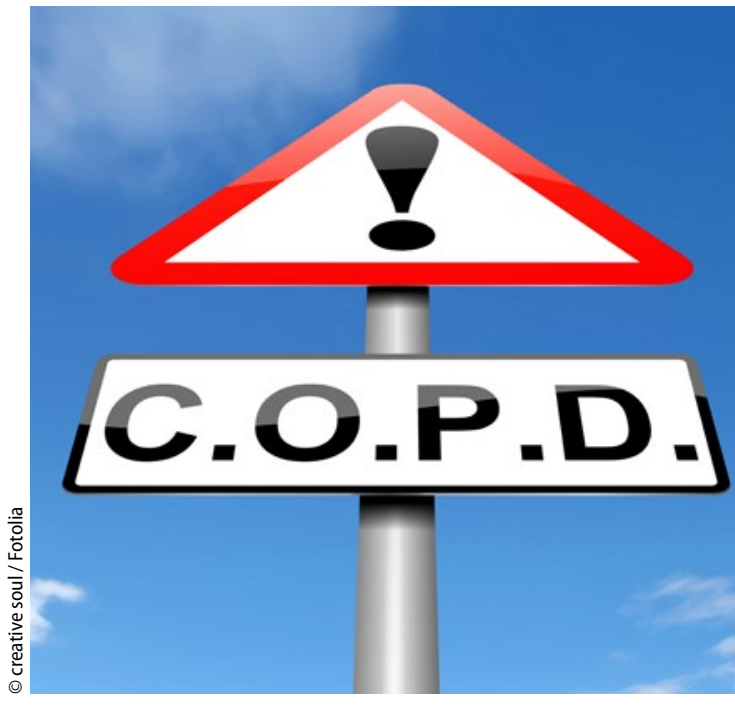

kann zudem ein einfach zu handhabender Inhalator die Adhärenz unterstützen.

Red.

\section{Beinulzera wirksam behandeln}

\section{Kompressionsbandagen-System für das Selbstmanagement}

\begin{abstract}
_ Die Kompressionstherapie ist in allen Stadien der chronisch-venösen Insuffizienz und beim Vorliegen eines Ulcus cruris venosum eine unverzichtbare Behandlungsoption [http://www.awmf. org/uploads/tx_szleitlinien/065-0021_ S2k_VTE_2016-01.pdf. Letzter Aufruf: 7. Mai 2016.] Kurzfristig führt die Kompression zu einer besseren Sauerstoffversorgung der Gewebe, langfristig zur Vermeidung des trophischen Gewebeumbaus und damit zur Abheilung der venösen Ulzera.
\end{abstract}

\section{Praktische Übungen}

Im Rahmen des Interprofessionellen Gesundheitskongresses 2016 in Dresden wurden praktische Übungen zur Durchführung der Wundreinigung und der Kompressionstherapie angeboten. Die
Teilnehmer erhielten u.a. die Möglichkeit, die Kompressionsstärke bei selbst gewickelten Verbänden mithilfe von Druckmesssonden zu überprüfen. Außerdem wurde das Management mit Kompressionsbinden-Systemen bzw. Wrap-Verbänden vorgestellt und praktisch geübt.

\section{Eigenverantwortliche Behandlung}

Ein Wrap-System, mit dem Patienten mit venösen Beinulzera sogar zu Therapeuten in eigener Sache werden können, ist circaid ${ }^{\circledast}$ juxtacures ${ }^{\circledast}$ : Die unelastische, atmungsaktive Versorgung kann von den Betroffenen selbstständig angelegt und auch genauso leicht wieder abgelegt werden.

Mithilfe des Built-In-Pressure-Systems (BPS) ist es auf einfache Weise möglich, die Versorgung bei sich verändernden
Beinumfängen - wie sie beispielsweise durch die rasche Ödemreduktion unter der Kompressionstherapie auftritt - individuell einzustellen bzw. nachzujustieren. Auf diese Weise gelingt es den Patienten ohne Schwierigkeiten, das vom Arzt verordnete Kompressionsniveau über die gesamte Tragedauer stabil zu halten.

Ein weiterer großer Vorteil des Bandagensystems ist, dass die Patienten ihre eigenen Schuhe tragen können, was ihre Mobilität im Alltag fördert. Die Wirksamkeit des Wrap-Systems in der Akuttherapie ist durch Studien mittlerweile belegt [Bender D et al. Veins and Lymphatics. 2013;2:30-36].

Dr. Silke Wedekind

- Workshop "Abschied vom Ulcus in drei Schritten", Interprofessioneller Gesundheitskongress 2016; Dresden, April 2016 (Veranstalter:medi) 